\title{
Information Visualization
}

- Biomedical Visualization, Visualisation on Built and Rural Environments \& Geometric Modelling and Imaging, IEETeL2017 -

\section{Edited by}

Ebad Banissi, Mark W. McK. Bannatyne, Fatma Bouali, Nuno Miguel Soares Datia, Georges Grinstein, Dennis Groth, Weidong Huang, Malinka Ivanova, Sarah Kenderdine, Minoru Nakayama, Joao Moura Pires, Muhammad Sarfraz, Marco Temperini, Anna Ursyn, Gilles Venturini, Theodor G. Wyeld, and Jian J. Zhang
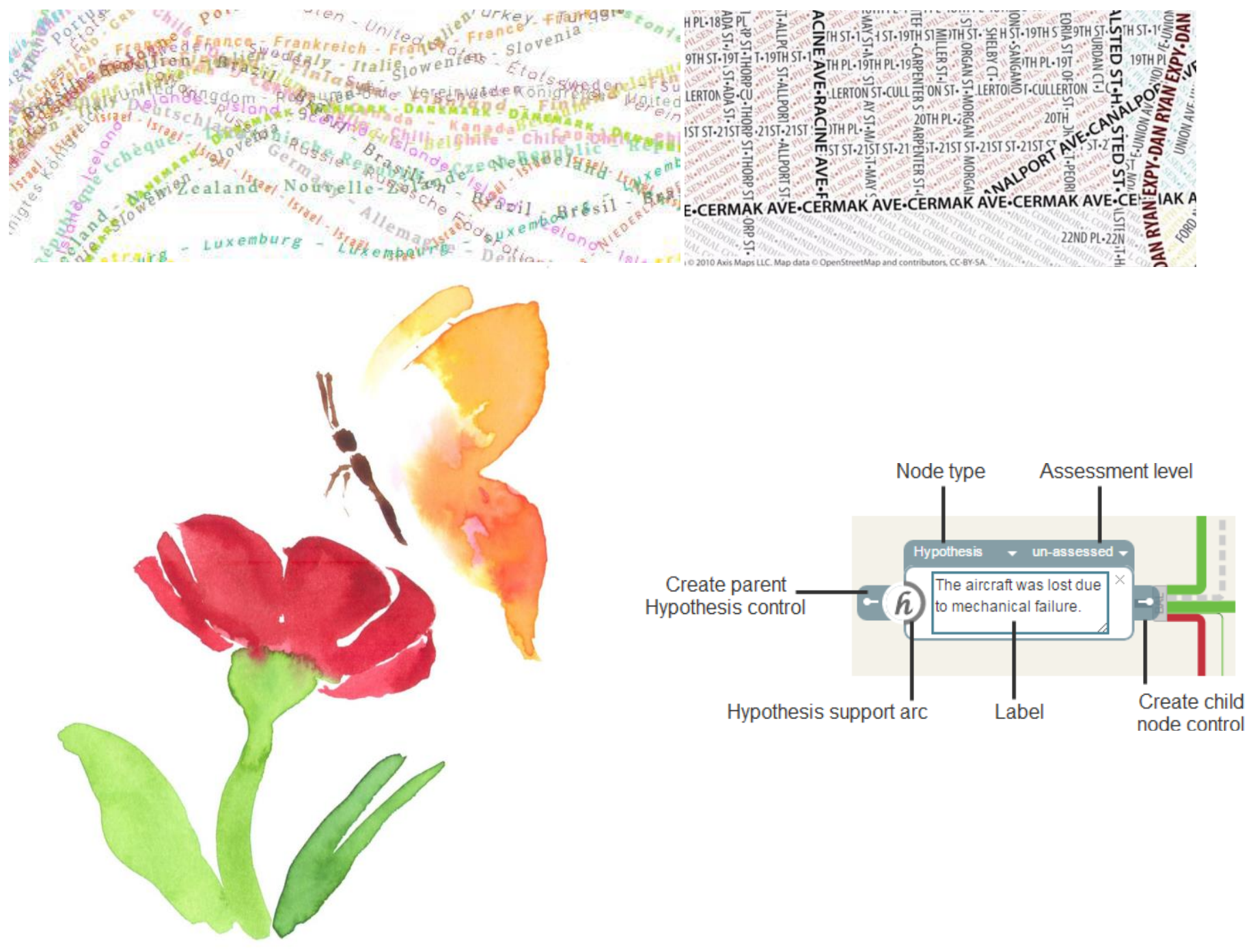

\section{Information Visualization}


- Biomedical Visualization, Visualisation on Built and Rural Environments \& Geometric Modelling and Imaging, IEETeL2017 -

\section{Edited by}

Ebad Banissi, Mark W. McK. Bannatyne, Fatma Bouali, Nuno Miguel Soares Datia, Georges Grinstein, Dennis Groth, Weidong Huang, Malinka Ivanova, Sarah Kenderdine, Minoru Nakayama, Joao Moura Pires, Muhammad Sarfraz, Marco Temperini, Anna Ursyn, Gilles Venturini, Theodor G. Wyeld, and Jian J. Zhang 


\section{Cover Image Credits}

\section{LEFT:}

' top

Microtext Line Charts

Richard Brath and Ebad Banissi

Uncharted Software Inc. (formerly Oculus Info Inc.) and London South Bank University

Figure 11. Top: text with halos to improve legibility. Bottom: over plotted text.

' bottom:

MediVis17Real-time Watercolours Simulation with Fluid Vorticity within Brush

Stroke

Roman Durikovic, Zuzana Palenikova

Comenius University Bratislava, FMFI - UK, Slovak Republic

Figure 1: Example of real life water color painting. Notice uneven spread of paint over paper's surface.

\section{RIGHT}

' top

\section{Microtext Line Charts}

Richard Brath and Ebad Banissi

Uncharted Software Inc. (formerly Oculus Info Inc.) and London South Bank University

Figure 3. Typographic map (axismaps.com)

\section{․ bottom}

Argument Mapper: Countering Cognitive Biases in Analysis with Critical (Visual) Thinking William Wright, David Sheffield, Stephanie Santosa

Uncharted Software Inc. (formerly Oculus Info Inc.)

Figure 6. Node editing controls. Clicking on a node, or hitting the enter key while a node is highlighted, allows a user to edit a node and its' properties. 


\title{
Information Visualization
}

\author{
- Biomedical Visualization, Visualisation on Built and Rural \\ Environments \& Geometric Modelling and Imaging, IEETeL2017 -
}

\section{Preface}

In the current information era, most aspects of life depend on and are driven by data, information, knowledge, user experience, and culture. The infrastructure of an information-dependent society relies on the quality of data, information and analysis of such entities for short to long term as well as past to future activities. Information Visualisation, Visual Analytics, Business Intelligence, Machine Learning and Application domains are just a few of the current state of the art developments that effectively enhance understanding of these driving forces. There are several key interdependent variables emerging that are becoming the focus of scientific activities, such as: Information and Data Science, an aspect that closely couples raw data (origin, autonomous capture, classification, incompleteness, impurity, filtering) and data scale to knowledge acquisition such that its dependencies on domain of application and its evolution steers the next generation of research conduct. Processing the relationship between these stages, from the raw data to visualisation, has added new impetus to the way these are understood and communicated. The tradition of use and communication by visualisation is deep rooted and helps us investigate new meanings for the humanities, history of art, design, human factors and user experience. Modern day computer assisted analytics and visualisation has added momentum in developing tools that exploit 2D and 3D metaphor-driven techniques within many applied domains. The techniques are developed beyond visualisation to simplify the complexities, to reveal ambiguity, and to work with incompleteness. The next phase of this evolving field is to understand uncertainty and risk analysis; how this uncertainty is built into the processes that exists in all stages of the process, from raw data to the knowledge acquisition stage.

This collection of papers on information visualization, compiled for the iV2017 conference, advocates that a new conceptual framework will emerge from information rich disciplines like the Humanities, Psychology, Sociology and Business as well as the science-rich disciplines. To facilitate this credence, iV2017 provides the opportunity to resonate with many international collaborative research projects as well as keynote lectures from distinguished speakers that channels the way this new framework conceptually, as well as practically has been realised. This year's theme is enhanced further by incorporating the 8th International workshop on Interactive Environments and Emerging Technologies for eLearning (IEETeL2017).

Joining us in this search are some 80 plus researchers who reflect and share a chapter of their thoughts with fellow researchers. The papers collected here reflect the vibrant state of Information Visualisation, Analytics, applications and results of the work of researchers, artists and professionals from more than 20 countries. It has allowed us to address the scope of visualisation from a much broader perspective. Each contributor to this conference has indeed added fresh perspective and thought, challenged our beliefs 
and encouraged further our adventure of innovation. I, on behalf of all editors of this book, am grateful to all the authors for sharing their valuable work with the information visualisation community.

Ebad Banissi

On behalf of editors 